\title{
MODELOS DE ESTUDIOS EN INVESTIGACIÓN APLICADA: CONCEPTOS Y CRITERIOS PARA EL DISEÑO
}

\author{
JORGE VEIGA DE CABO*, ELENA DE LA FUENTE DÍEZ**, \\ MARTA ZIMMERMANN VERDEJO***.
}

(*) Escuela Nacional de Medicina del Trabajo.

Instituto de Salud Carlos III. Madrid.

(**) Servicio de Obstetricia y Ginecología.

Consorcio Hospital General Universitario. Valencia.

$(* * *)$ Servicio de Salud Laboral.

Dirección General de Salud pública y Alimentación. Comunidad de Madrid.

\section{RESUMEN}

Se pretende dar una noción general de los conceptos básicos a la hora de aproximarse a un diseño de estudios epidemiológicos en investigación en ciencias de la salud, describiendo y clasificando los estudios, analizando los principales criterios que han de tenerse en cuenta a la hora de diseñar un estudio y los principales problemas con los que nos podemos encontrar desde el punto de vista práctico.

\section{PALABRAS CLAVES}

Estudios epidemiológicos, estudios analíticos, estudios experimentales, estudios descriptivos, estudios de casos y controles, estudios de cohortes, falacia ecológica, sesgos, diseño.

\begin{abstract}
The aim of the article is offer a general notion about the main concepts to design epidemiologist studies in health sciences research, describing and classifying the different kind studies, analyzing the criteria for design and exploring the main problems we can find in epidemiologist research studies.
\end{abstract}

\section{KEY WORDS}

Epidemiologist studies, analytic studies, experimental studies, descriptive studies, cases control studies, cohort studies, fallacy, bias. 


\section{INTRODUCCIÓN}

Conceptualmente podemos definir la epidemiología como la Ciencia del razonamiento y del método usados en medicina y en otras ciencias, aplicadas a la descripción de los fenómenos de salud, a la explicación de su etiología y a la búsqueda de método de intervención más eficaces.

Los modelos de estudios que se aplican en epidemiología son diferentes, en la medida en que también lo son los objetivos que se persiguen. La observación (suministrada, por ejemplo, a partir de los sistemas de vigilancia que recogen información continua sobre morbilidad, o aquella obtenida a partir procedimientos más simples como las encuestas) es una actitud inicial que nos permitirá la detección y descripción de los problemas de salud que aparecen en una comunidad.

Partiendo de esta base conceptual, podremos plantearnos una primera hipótesis. Posteriormente, y dependiendo del tipo de problema que tenemos que investigar o del tiempo, o incluso del presupuesto del que dispongamos, tendremos que elegir el tipo de estudio epidemiológico más apropiado para responder a nuestras preguntas.

Antes de iniciar cualquier tipo de investigación, tenemos que tener muy claro cuales son nuestros objetivos, la población que queremos estudiar, las variables que queremos recoger, los métodos para hacerlo, el tiempo que nos llevará cada fase de la investigación, los medios técnicos y humanos que necesitaremos, entre otros aspectos básicos.

En teoría toda investigación va asociada a una verificación de una pregunta mediante la experimentación. Sin embargo, en medicina, donde el objeto de investigación es el ser humano, no es siempre posible la experimentación, por ello han proliferado y se han consolidado otros tipos de diseño basados en la observación. Este planteamiento nos lleva directamente a la primera división entre los distintos tipos de estudios que se pueden presentar: los estudios experimentales y los estudios observacionales.

Los estudios observacionales pretenden describir un fenómeno dentro de una población de estudio y conocer su distribución en la misma. En este tipo de estudios, no existe ninguna intervención por parte del investigador, el cual se limita a medir el fenómeno y describirlo tal y como se encuentra presente en la población de estudio.

A su vez, los estudios observacionales pueden ser Descriptivos o Analíticos, en función de los objetivos que persigan y el diseño para alcanzarlos. En los estudios descriptivos, el investigador se limita a medir la presencia, características o distribución de un fenómeno dentro de la población de estudio como si de un corte en el tiempo se tratara. En los estudios analíticos, el investigador pretende relacionar causalmente algún factor de riesgo o agente causal con un determinado efecto, es decir, pretende establecer una relación causal entre dos fenómenos naturales.

En los estudios Experimentales, el investigador también trata de estudiar algún factor desconocido y sus efectos en el tiempo, pero al contrario de lo que ocurre en los estudios observacionales analíticos, que reproducen el fenómeno de forma natural, en los estudios experimentales el investigador define cada una de las características de los grupos, asignando a un grupo de estudio el tratamiento, tóxico, prueba diagnóstica, factor de riesgo, o lo que se pretenda estudiar, y al grupo control el placebo o su equivalente.

Entre estos estudios se encuentran los denominados ensayos controlados, que son experimentos en los que los sujetos son asignados de manera aleatoria, a grupos, generalmente denominados "grupo de estudio" y "grupo control", para recibir, o no recibir, un procedimiento, maniobra o intervención, preventivos o terapéuticos, de carácter experimental.

Los estudios experimentales, cuando éstos están bien diseñados y no existen fuentes de error o sesgos, son considerados los diseños más potentes para la comprobación de hipótesis causales.

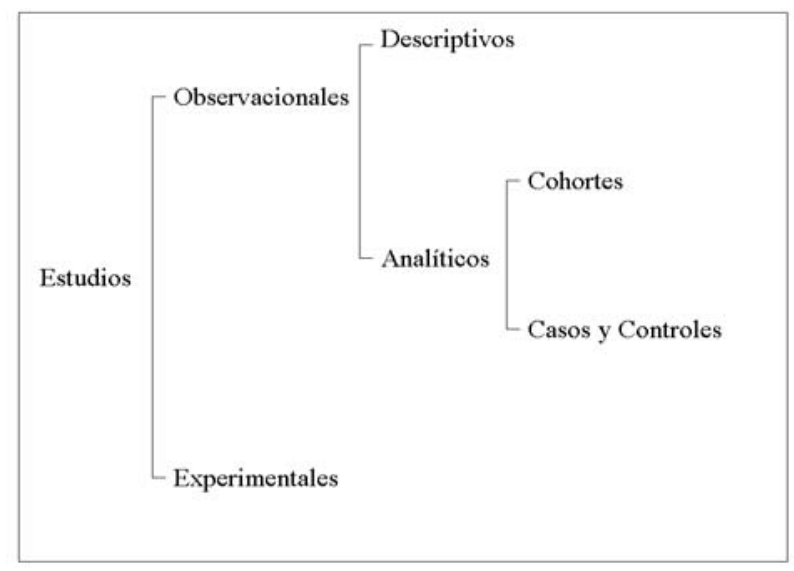

Este tipo de estudios son poco frecuentes en el campo sanitario en el que nos solemos desenvolver, siendo más propios en el ámbito del a investigación básica, investigación en laboratorios o ensayos clínicos controlados, por poner algunos ejemplos, y 
requieren una serie condicionantes de garantías éticas y autorización del comité de investigación, razones por las que no serán objeto nuestro de estudio en profundidad.

\section{ESTUDIOS OBSERVACIONALES}

Dentro de los estudios observacionales, podríamos diferenciar dos tipos de estudios, según el objetivo que persigan, y por tanto, su diseño. Nos referimos a estudios Descriptivos y estudios Analíticos.

\section{1.- Estudios Descriptivos}

En los estudios descriptivos, el investigador se limita a medir la presencia, características o distribución de un fenómeno en una población en un momento de corte en el tiempo, tal sería el caso de estudios que describen la presencia de un determinado factor ambiental, una determinada enfermedad, mortalidad en la población, etc., pero siempre referido a un momento concreto y sobre todo, limitándose a describir uno o varios fenómenos sin intención de establecer relaciones causales con otros factores. Por tanto, la principal característica de los estudios descriptivos es que se limitan simplemente a "dibujar" el fenómeno estudiado, sin pretender establecer ninguna relación causal en el tiempo con ningún otro fenómeno, para lo que necesitaríamos recurrir a un estudio analítico.

La primera finalidad de estos estudios, como bien indica su nombre, es describir la frecuencia y las características más importantes de un problema de salud en una población. Su segunda función es proporcionar datos sobre los que basar hipótesis razonables.

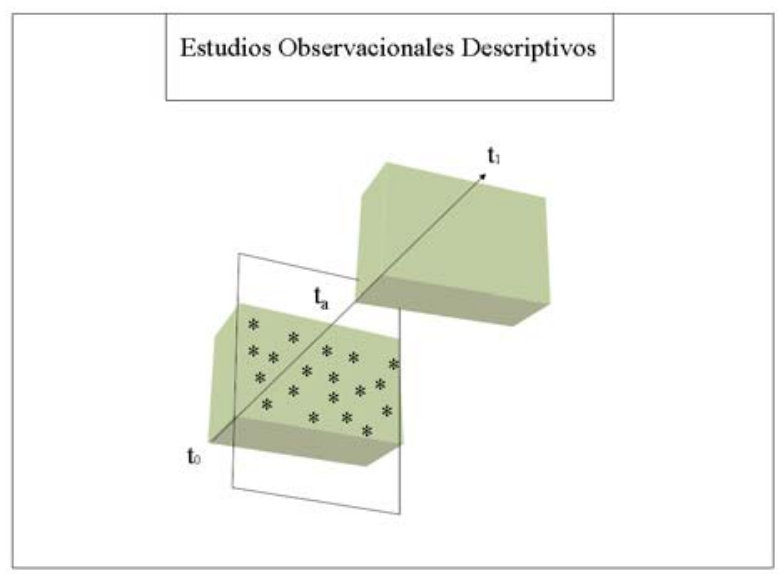

De esta forma, un estudio de tendencias de mortalidad en un periodo determinado, hemos de considerarlo como un estudio descriptivo, pues aunque describa el fenómeno y su evolución dentro de un espacio temporal, el estudio se limita a la descripción del fenómeno dentro de unas coordenadas temporales, pero sin establecer relación causal con ningún factor concomitante en el tiempo. En este caso, el periodo de tiempo más o menos amplio en el que se encuadre el estudio, se considera como si de corte en el tiempo se tratase.

La característica principal que lo define como estudio observacional descriptivo y lo diferencia de un estudio observacional analítico es que en este último, su objetivo y su diseño se centran, como se ha mencionado, en buscar alguna relación causaefecto entre dos fenómenos que se relacionan a lo largo del tiempo, como veremos después.

Algunos ejemplos que podemos citar de estudios descriptivos, serían los estudios de incidencia, de prevalencia, de evaluación de riesgos, de encuestas poblacionales, por citar los más corrientes, y constituyen, en su mayor parte, el modelo de estudio más frecuente con el que nos encontremos dentro de nuestro ámbito de actividad sanitaria, lo que no quita que con relativa frecuencia podamos encontrarnos, $o$ nos interese diseñar algún otro tipo de estudios.

En numerosas ocasiones, por ejemplo al realizar una encuesta en la que se ha recogido gran cantidad de variables, el investigador, queda abrumado por el exceso de información. Así, en la fase de análisis a veces olvida que se trata de un "dibujo" y comienza a interpretar las asociaciones estadísticas entre una exposición o factor y un estado de salud, como si de relaciones causa-efecto se tratase. En otras palabras, una característica que define estos estudios es la falta de secuencia temporal entre la medición de un factor de riesgo y un hipotético efecto, por tanto, es extremadamente complicado corroborar hipótesis causales.

Esto se suele agravar dada la facilidad con que actualmente pueden realizarse análisis estadísticos. En general, y en los estudios descriptivos en particular, uno puede obtener cientos de asociaciones "estadísticamente significativas" sin que esto, desde el punto de vista científico, signifique nada en absoluto.

Otras veces, los investigadores noveles sucumben ante una irresistible "corazonada" e incurren en un error, también muy frecuente, denominado "falacia ecológica". 
Para entender la falacia ecológica vamos a recurrir a un ejemplo. Imaginemos que queremos estudiar los accidentes laborales ocurridos durante el último año en una determinada empresa. Para ello llevamos a cabo un estudio de incidencia de accidentes, es decir, un estudio descriptivo de la patología durante el tiempo de estudio, un año. Supongamos que el resultado de nuestro estudio es que encontramos una elevada tasa de incidencia de accidentes, y de pronto se nos ocurre que detrás de esta alta tasa de accidentes pudiera esconderse un elevado nivel de estrés en los trabajadores.

A partir de ese momento decidimos ampliar nuestro estudio y le incorporamos un análisis de la proporción de trabajadores que sufren un elevado nivel de estrés, y curiosamente, nos encontramos que el porcentaje de trabajadores de la empresa con nivel de estrés alto muestra también cifras elevadas.

Nuestra intención podría ser asociar los hallazgos y establecer relación entre ellos, por el hecho de que se encuentran presentes en la misma población, determinando una relación causal entre el elevado nivel de estrés de los trabajadores y la alta proporción de accidentes detectados en la empresa, pero si así lo hiciéramos, estaríamos cometiendo un grave error metodológico.

La falacia ecológica se produce desde el momento en que, aún realizándose el estudio de los accidentes y del estrés en una misma población cerrada, se trata de dos estudios observacionales diferentes, aunque se encuentren solapados. Si utilizamos dos estudios descriptivos para sacar conclusiones conjuntas de los resultados de cada uno de ellos, nunca podemos estar seguros de que el subconjunto de población que se encuentra afectada por un elevado nivel de estrés, se corresponda exactamente o incluso aproximadamente, con el subconjunto de población en la que detectamos los accidentes de trabajo.

A veces, las observaciones epidemiológicas descriptivas proporcionan el primer aviso de los, hasta el momento, riesgos desconocidos. Pero sin duda la mayor virtud de los estudios descriptivos reside en su utilidad en la planificación y en su potencial como generadores de hipótesis (que deberán ser corroboradas con estudios más potentes).

En resumen, siempre que pretendamos estudiar una asociación causal, tendremos que recurrir a un diseño que nos permita relacionar la causa y el efecto en cada uno de los individuos estudiados, con lo que al menos, podemos tener la certeza de que la hipotética causa y el supuesto efecto se encuentran relacionados individualmente y se corresponden con la misma población, por lo que debemos recurrir a los estudios analíticos.

\section{2.- Estudios Analíticos}

Los estudios Analíticos se caracterizan porque pretenden "descubrir" una hipotética relación entre algún factor de riesgo y un determinado efecto, es decir, pretenden establecer una relación causal entre dos fenómenos naturales.

En este tipo de estudios, es esencial entender que para que prevalezca su condición de estudio observacional, han de cumplir la premisa de que el investigador no establece ningún tipo de intervención en los grupos de estudio, los cuales se someten a las leyes naturales de evolución desde que interviene la causa hasta que se produce el hipotético efecto resultante.

Los estudios analíticos requieren un diseño especial y más cuidadoso que los estudios descriptivos, sobre todo en lo que se refiere a los sistemas de control de sesgos y de factores de confusión, que de no establecerse desde su diseño inicial de forma correcta podrían invalidar los resultados del estudio.

Si bien decíamos que los estudios descriptivos nos muestran una fotografía estática de un fenómeno concreto, bien sea el factor de riesgo, como por ejemplo un estudio de evaluación de riesgos, o del efecto, como pudiera ser un estudio de incidencia o prevalencia, los estudios analíticos nos dan una visión dinámica del proceso salud-enfermedad y las posibles relaciones causales definidas en el tiempo desde que se hace presente el agente causal hasta que aparece la enfermedad, por lo que los estudios Analíticos nos vendrían a ofrecer una visión dinámica similar a una película en la que podemos relacionar el desenlace con diferentes escenas previas de la misma.

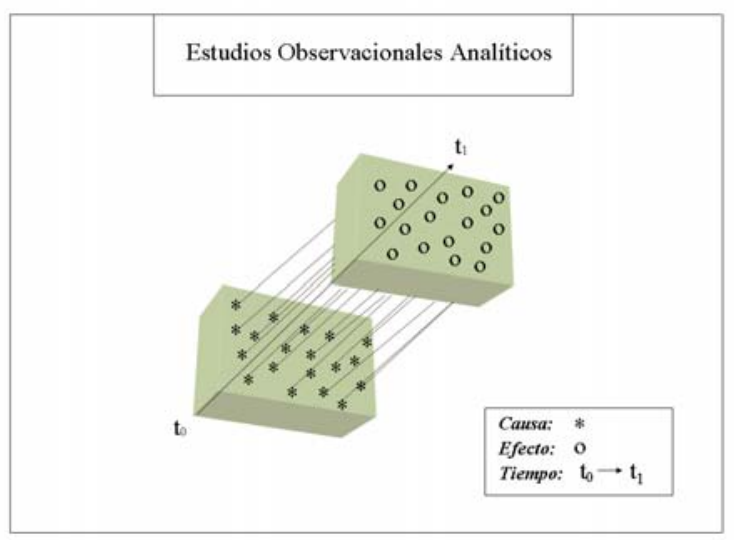


Traducido al marco conceptual del tipo de estudio de que se trata, los estudios analíticos contarían con un diseño que nos permitirá hacer el seguimiento dentro de un proceso evolutivo natural, desde la aparición de los hipotéticos factores causales hasta que culmine con la aparición del supuesto efecto, lo que implica asentar unos criterios básicos.

En primer lugar, debemos tener claro que desde que actúa un determinado factor causal hasta que se produce el efecto, en su caso, la enfermedad, siempre transcurre un tiempo (tiempo de latencia), que en unos casos puede ser corto y en otros más o menos largo, pero ambos fenómenos se encuentran relacionados en el tiempo definido entre la presencia o aparición de cada uno de ellos.

Este concepto, además centrar la relación causal como un proceso dinámico dentro de un espacio y tiempo definidos, nos permite determinar otra característica tan obvia como importante, la causa ha de preceder siempre en el tiempo al efecto.

El segundo lugar, la mayoría de los fenómenos naturales, y por tanto, entendiendo la enfermedad como uno de ellos, se producen como resultado de la confluencia de varios factores causales y difícilmente en circunstancias normales podremos achacar a un único fenómeno que pueda ser el causante en exclusiva de la aparición un efecto. Así, debemos entender la dinámica salud-enfermedad como una relación causa-efecto de características multicausales, en las que las diferentes causas que intervienen, se comportan todas ellas como "causas componentes" dentro de una cadena. Algunas de estas causa pueden manifestarse con mayor o menos fuerza etiológica que otras y por tanto, unas se comportan con un mayor peso "determinante" que otras, incluso alguna (o varias de ellas), podríamos considerarla como "causa necesaria", siendo siempre obligatoria su presencia dentro de la cadena para que aparezca el efecto.

El tercer concepto a tener en cuenta, es que siempre que nos planteemos el objetivo de establecer una relación causal entre un determinado factor como hipotético causante de un efecto determinado, no podremos limitarnos a estudiar únicamente un grupo de población en el que supuestamente se encuentren presentes los dos fenómenos del estudio (causa y efecto), tendremos que diseñar un tipo de estudio que contemple la posibilidad de comparar los resultados de este grupo con otro que a modo de control, incluya individuos que pudieran no presentar relación con alguno de los factores estudiados (causa o efecto), realizando un seguimiento individual de cada grupo para analizar conjuntamente los resultados en busca de posibles diferencias.

Volviendo a nuestro ejemplo del estudio sobre accidentes de trabajo en una empresa y su posible relación con la carga de estrés en los trabajadores, ya hemos desestimado abordarlo mediante un solapamiento de estudios descriptivos, como hemos visto. Parece más lógico si tomásemos a la población que ha sufrido accidentes laborales, y estudiásemos en ellos su relación con el nivel de estrés que presentaban inmediatamente antes de que se produjera el mismo. De esta forma estamos seguros de que el nivel de estrés y el accidente se encuentran medidos en el mismo subgrupo de trabajadores de la empresa y evitaríamos el riesgo de incurrir en una falacia ecológica.

Pero si diseñamos nuestro estudio sobre lo que ocurre exclusivamente en la población de trabajadores que han sufrido algún tipo de accidente laboral durante el último año y pretendemos relacionar las dos variables desde el punto de vista causal, tendríamos certeza que nos encontramos midiendo la relación entre la carga de estrés dentro del la población de trabajadores accidentaos, pero desconoceríamos que relación que pudiéramos encontrar entre la carga de estrés y la población no accidentada.

Si planteamos el estudio de esta forma, podríamos encontrarnos con unos resultados que apoyasen nuestra hipótesis, tras observar que los trabajadores accidentados presentaban una elevada carga de estrés laboral, previo al accidente, pero ¿podríamos concluir que la causa de que se produzcan accidentes laborales es el estrés?, ¿estaríamos en condiciones de mantener esta tesis si estudiado el grupo de trabajadores no accidentado encontrásemos que el nivel de estrés durante el mismo periodo fuera similar al encontrado entre los trabajadores accidentados?, incluso, ¿a que conclusión podríamos llegar si el nivel de estrés de la población no accidentada fuera superior a la de los trabajadores accidentados?, ¿no podríamos estar en este caso ante una situación en la que el estrés podría comportarse como un supuesto factor protector frente al riesgo de sufrir algún accidente de trabajo?

Lo que nos está ocurriendo con este tipo de diseño es que, aún siendo correcto para controlar la falacia ecológica, le falta un grupo control que nos permita saber que está ocurriendo en la otra población, la no accidentada, de forma que no podemos asegurar de forma consistente la relación causal, no porque no exista, que podría existir en base a los resultados, sino porque no tenemos información 
suficiente para poder asegurar que realmente lo sea. Hemos analizado lo que pasa en un sólo grupo de población, los trabajadores accidentados, pero desconocemos totalmente lo que ocurre en la población no accidentada en relación a la carga de estrés. En definitiva, el simple hecho de no poder responder estas preguntas con certeza nos sumerge en un mar de incertidumbres y nos impide alcanzar cualquier conclusión consistente.

Para solucionar este problema, deberíamos diseñar un estudio de forma que además de analizar la relación entre los trabajadores accidentados y su exposición a el estrés previo, incluyamos el mismo análisis un grupo que actúe como "control", en este caso, compuesto por los trabajadores que no han sufrido accidentes, y estudiemos en ellos también su relación con nuestro hipotético agente causal, de forma, que comparando posteriormente los resultados en cada uno de los grupos (accidentados y no accidentados), tengamos información suficiente como para comprobar, utilizando procedimientos estadísticos adecuados, que la presencia del factor de riesgo (estrés) se presenta de forma significativamente diferente entre los dos grupos de población estudiados (accidentados y no accidentados).

Para ello podemos recurrir a dos modalidades de estudios analíticos: Estudios de Cohortes (habría que diferenciarlos de los estudios de corte, los cuales se refieren en su mayor parte a estudios realizados en un determinado momento, o corte en el tiempo) y los estudios de Casos y Controles.

\section{1.- Estudios de Cohortes}

En este tipo de estudios se establecen dos cohortes, una se corresponde con la cohorte de estudio y estará compuesta por la población expuesta al hipotético factor de riesgo, y la otra, se comporta como cohorte control, compuesta por individuos que no se encuentren expuestos al hipotético factor de riesgo. Establecidas las cohortes, se realiza un seguimiento en el tiempo de cada una de ellas (población expuesta y población no expuesta), estudiando el comportamiento de la cada una en cuanto a la aparición del efecto o enfermedad o desenlace (incidencia). También se les denomina prospectivos por que van "hacia adelante" desde la exposición hasta un efecto.

De esta forma, de ser cierta nuestra hipótesis, cabría esperar en esta cohorte de expuestos una tasa (incidencia) importante de afectados (o al menos comparativamente superior a la encontrada en los no expuestos).
La cohorte de los no expuestos, actuaría como sistema control, de forma que de ser cierta la hipótesis, cabría esperar una baja tasa de afectados (o al menos comparativamente inferior a la encontrada en los expuestos).

En cierto modo, desde el momento en que establecemos una cohorte control, nos encontraremos en condiciones poder ofrecer unos resultados consistentes con la hipótesis causal de trabajo. Esto es cierto, siempre que el diseño del estudio sea impecable, el grupo de expuestos y no expuestos no difieran en otras variables predictoras del desenlace y no hayamos introducido cualquier otra fuente de error en la medición del efecto o en el seguimiento.

\section{2.- Estudios de Casos y Controles}

En los estudios de casos y controles se elige un grupo de individuos que tienen una enfermedad determinada (casos), y otro en el que está ausente. Ambos grupos se comparan respecto a una exposición que se sospecha que está relacionada con dicha enfermedad. La función del grupo control es precisamente estimar la proporción de exposición esperada en un grupo que no tiene la enfermedad.

Lo estudios de Casos y Controles tiene en común con los estudios de Cohortes que también se establecen dos poblaciones. La diferencia estriba en que, en los estudios de Casos y Controles las poblaciones de estudio se establecen en función de la presencia o no del efecto o enfermedad, es decir, el grupo de los casos estaría compuesto por los individuos que presentan la enfermedad o efecto estudio, y el grupo Control por la población que no padece la enfermedad.

En este caso, el estudio no requiere de un seguimiento, sino que su enfoque es retrospectivo, "hacia atrás", para estudiar la relación, contacto o exposición previa de cada uno de los grupos (enfermos y no enfermos) con uno o varios hipotéticos factores de riesgo. Generalmente para la reconstrucción de la historia de exposición se recurre a encuestas, revisión de historias clínicas, analíticas, exploraciones, etc.

En definitiva, en un estudio de casos y controles lo que se mide es si la frecuencia del factor de riesgo entre nuestros casos enfermos es superior a la observada en nuestros controles no enfermos.

Aunque el abordaje parece simple, se sorprendería de las enormes dificultades asociadas a estos diseños. Así, a la hora de diseñar un estudio de casos 
y controles, es muy recomendable ser especialmente cuidadoso para no incurrir en las numerosas fuentes de error que amenazan la validez del estudio. Los errores más frecuentes en estos estudios se cometen en el procedimiento de selección de los controles (deben proceder de la misma población donde se han extraído los casos y ser lo más perecidos posible a éstos, excepto en padecer o no la enfermedad) y durante la recogida de información para reconstruir la historia de exposición de ambos grupos.

En resumen, en los estudios de Cohortes se parte desde la exposición al factor de riesgo para llegar a la enfermedad y en los estudios de Casos y Controles se parte de la enfermedad para intentar establecer una relación causal previa con el factor de riesgo.

Como es lógico, los estudios de Cohortes, al contar con un diseño en el que, por lo general, se basa en el seguimiento de la evolución natural de la dinámica salud-enfermedad, suelen tratarse de estudios más fiables que los estudios de Casos y Controles, pero a la vez suelen ser más costosos, no sólo por el tiempo necesario de dedicación al estudio durante el seguimiento, sino también en lo que se refiere al resto de recursos.

Por el contrario, los estudios de Casos y Controles suelen ser más rápidos y económicos, aunque por lo general, requieren especial cuidado en establecer desde el diseño los mecanismos necesarios para el control de sesgos y factores de confusión, que no es objeto de este artículo. Por esta razón, se suelen tomar dos tipos de medidas que ayudan a incrementar la fiabilidad de resultados, por una parte es habitual establecer un sistema "apareado" de selección de casos y controles. Esto supone seleccionar la población de casos e ir estableciendo la población de controles lo más parecida a los casos excepto en la condición que define al control, el "no padecer la enfermedad objeto de estudio".

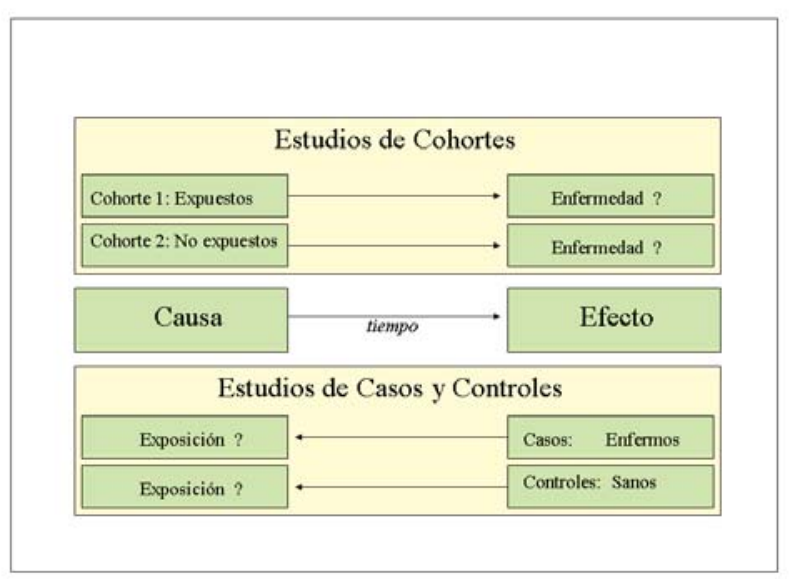

\section{2.- Otras formas de clasificación de los estu- dios}

\section{1.- Estudios Longitudinales y Transversales}

Además de la clasificación que hemos visto, existe también la posibilidad de clasificar los estudios desde el punto de vista de su dimensión espacio-temporal. Así, los estudios Longitudinales se corresponderían con aquellos en los que se presenta una temporalidad como base del mismo. Dentro de los estudios descriptivos, serían longitudinales los estudios de tendencias o aquellos que establecen un periodo de tiempo como base del estudio. Los estudios transversales se corresponden con los estudios de corte en el tiempo.

Los estudios analíticos se consideran longitudinales por definición, pues el criterio para que un estudio sea calificado como longitudinal, radica en la base conceptual que subyace en el estudios, de forma que en el caso de los estudios, tanto de Cohortes como de Casos y Controles, la base del estudio se establece en el tiempo real o implícito establecido entre el momento en que actúa un factor de riesgo o agente causal y el que se muestra patente el efecto o enfermedad.

\section{2.- Estudios Prospectivos y Retrospectivos}

Este tipo de clasificación se establece en cuanto a la dimensión longitudinal de los estudios, por lo que se pueden excluir directamente los estudios de corte o transversales.

Los criterios de prospectivo o retrospectivo, se refieren principalmente al planteamiento de la dirección en el tiempo del estudio, progresiva (hacia delante) o regresiva (hacia atrás) en el tiempo desde el momento en que se inicia el estudio. En el caso de los estudios descriptivos longitudinales, podemos encontrar estudios prospectivos, cuando una vez establecido el inicio del estudio se realiza un seguimiento de la población en el tiempo, y lo consideraríamos retrospectivo (en la mayoría de los casos), cuando analizamos una tendencia de cualquier fenómeno que haya acontecido en una población con anterioridad al inicio del estudio.

Dentro de los estudios analíticos, los estudios de Casos y Controles se considerarán retrospectivos, pues el momento del inicio del estudio se establece una vez se ha hecho presente el efecto o enfermedad, y se retrocede en el tiempo para analizar exposiciones previas al factor de riesgo o causas. 
Conceptualmente, los estudios de Cohortes deberíamos considerarlos prospectivos, pues el criterio principal que los define es su dirección desde la exposición al factor de riesgo hacia la aparición de la enfermedad, lo que ocurre siempre con carácter posterior a al exposición. Ahora bien, si nos atenemos al criterio del momento en que se inicia el estudio, pue-

\section{BIBLIOGRAFÍA}

1. Ahlbom A, Novell S. Fundamentos de epidemiología. Siglo XXI Editores. Madrid 1995.

2. Argimon Pallas JM, Jiménez Villa J. Métodos de investigación. Mosby/Doyma. Barcelona 1994.

3. Argimon Pallas JM. Métodos de investigación clínica y epidemiológica. Elsevier España; 2004.

4. Hernández-Aguado I, Gil A, Delgado M y Bolumar F. Manual de epidemiología y salud pública para licenciaturas y diplomaturas en ciencias de la salud. Madrid: Panamericana; 2005. den considerarse retrospectivos aquellos estudios de cohortes en los que la enfermedad objeto de estudio se haya producido tiempo antes del momento de iniciación del estudio. Por ello a este último diseño "particular" se le denomina cohorte retrospectiva, cuyas peculiaridades merecerían ser ampliamente comentadas en un artículo específico.
5. Hernberg S. Introducción a la epidemiología ocupacional. $1^{\mathrm{a}}$ edición. Ediciones Diaz de Santos. Madrid 1995 .

6. Jenicek M. Epidemiología: la lógica de la medicina moderna. Barcelona: Masson; 1996.

7. Rothman K.J. epidemiología Moderna. Ediciones Díaz de Santos. Madrid 1987. 